\title{
Stacking Domains and Dislocation Networks in Marginally Twisted Bilayers of Transition Metal Dichalcogenides
}

\author{
V. V. Enaldiev, ${ }^{1,2,3}$ V. Zólyomi, ${ }^{4,1,2}$ C. Yelgel®, ${ }^{5,1,2}$ S. J. Magorrian, ${ }^{1,2}$ and V. I. Fal'ko $\circledast^{1,2,6}$ \\ ${ }^{1}$ National Graphene Institute, University of Manchester, Booth St. E. Manchester M13 9PL, United Kingdom \\ ${ }^{2}$ School of Physics and Astronomy, University of Manchester, Oxford Road, Manchester M13 9PL, United Kingdom \\ ${ }^{3}$ Kotel'nikov Institute of Radio-engineering and Electronics of the Russian Academy of Sciences, \\ 11-7 Mokhovaya St, Moscow 125009, Russia \\ ${ }^{4}$ Hartree Centre, STFC Daresbury Laboratory, Daresbury WA4 4AD, United Kingdom \\ ${ }^{5}$ Recep Tayyip Erdogan University, Department of Electricity and Energy, Rize 53100, Turkey \\ ${ }^{6}$ Henry Royce Institute for Advanced Materials, University of Manchester, Manchester M13 9PL, United Kingdom
}

(Received 28 November 2019; accepted 13 April 2020; published 20 May 2020)

\begin{abstract}
We apply a multiscale modeling approach to study lattice reconstruction in marginally twisted bilayers of transition metal dichalcogenides (TMD). For this, we develop density functional theory parametrized interpolation formulae for interlayer adhesion energies of $\mathrm{MoSe}_{2}, \mathrm{WSe}_{2}, \mathrm{MoS}_{2}$, and $\mathrm{WS}_{2}$, combine those with elasticity theory, and analyze the bilayer lattice relaxation into mesoscale domain structures. Paying particular attention to the inversion asymmetry of TMD monolayers, we show that $3 R$ and $2 H$ stacking domains, separated by a network of dislocations develop for twist angles $\theta^{\circ}<\theta_{\mathrm{P}}^{\circ} \sim 2.5^{\circ}$ and $\theta^{\circ}<\theta_{\mathrm{AP}}^{\circ} \sim 1^{\circ}$ for, respectively, bilayers with parallel (P) and antiparallel (AP) orientation of the monolayer unit cells and suggest how the domain structures would manifest itself in local probe scanning of marginally twisted $\mathrm{P}$ and AP bilayers.
\end{abstract}

DOI: 10.1103/PhysRevLett.124.206101

Layer-by-layer assembly of van der Waals (vdW) heterostructures of two-dimensional crystals became a popular method of creating new hybrid materials [1]. The underlying physics of optoelectronic properties of such systems includes the interlayer hybridization of electronic states of the layers and the superlattice effects, produced by the periodic moire patterns characteristic to pairs of mutually twisted or slightly incommensurate lattices. Such effects have been extensively investigated in graphene-hBN heterostructures [2-6] and twisted graphene bilayers [7-9], and these studies have identified two distinct structural forms of bilayers. One corresponds to larger twist angles, $\theta$, and a stronger lattice mismatch, $\delta$, for which the periodic variation of local stacking of the atoms (moiré pattern with period $\ell=a / \sqrt{\delta^{2}+\theta^{2}}$ ) is due to the superposition of rigid crystalline lattices of the two layers [2-9]. The other, "marginally twisted bilayers" [10] regime is peculiar to a very small misalignment in graphene bilayers, which reconstruct into large-area Bernal stacking domains, separated by networks of domain walls [11-18].

Published by the American Physical Society under the terms of the Creative Commons Attribution 4.0 International license. Further distribution of this work must maintain attribution to the author(s) and the published article's title, journal citation, and DOI.
Beyond graphene and hBN, moiré superlattice effects have been observed in $M X_{2} / M^{\prime} X_{2}^{\prime}$ heterostructures of transition metal dichalcogenides (TMD) [19-25], and it has been suggested theoretically [26-28] that twisted bilayers of TMD can undergo lattice reconstruction. Here, we determine parametric conditions for the formation of and the types of domain structures in twisted TMD homo- and heterobilayers, enriched by the lack of inversion symmetry of the individual 2D crystals. Different domain wall networks form for bilayers with parallel $(\mathrm{P})$ and antiparallel (AP) orientations of unit cells in the two layers (shown on top of Fig. 2), and we find crossover angles, $\theta_{\mathrm{AP}}^{\circ} \sim 1.0^{\circ}$ and $\theta_{\mathrm{P}}^{\circ} \sim 2.5^{\circ}$, for the marginality of the twist and, then, discuss how the resulting domain structures can be observed in scanning tunneling experiments.

In this study we employ the multiscale modeling: a combination of density functional theory (DFT) leading to interpolation formulae for adhesion energy, $W_{\mathrm{P} / \mathrm{AP}}\left(\boldsymbol{r}_{0}, d\right)$, between the layers at a distance $d$ from each other and lateral offset $\boldsymbol{r}_{0}$, and elasticity theory for the lattice relaxation [27]. We perform this analysis for small misalignment angles, $\theta \ll 1$ (i.e., $\theta^{\circ}<5^{\circ}$ ), and lattice mismatch $\delta \ll 1$. In this case energetics of local stacking can be described in terms of a lateral offset, $\boldsymbol{r}_{0}(\boldsymbol{r})=\theta \hat{z} \times \boldsymbol{r}+$ $\delta \boldsymbol{r}+\boldsymbol{u}^{(t)}-\boldsymbol{u}^{(b)}$, between two aligned commensurate lattices, which varies across the moiré supercell and includes the effect of local deformations, $\boldsymbol{u}^{(b / t)}(\boldsymbol{r})$, in the bottom and top layers. This multiscale approach enables us to 


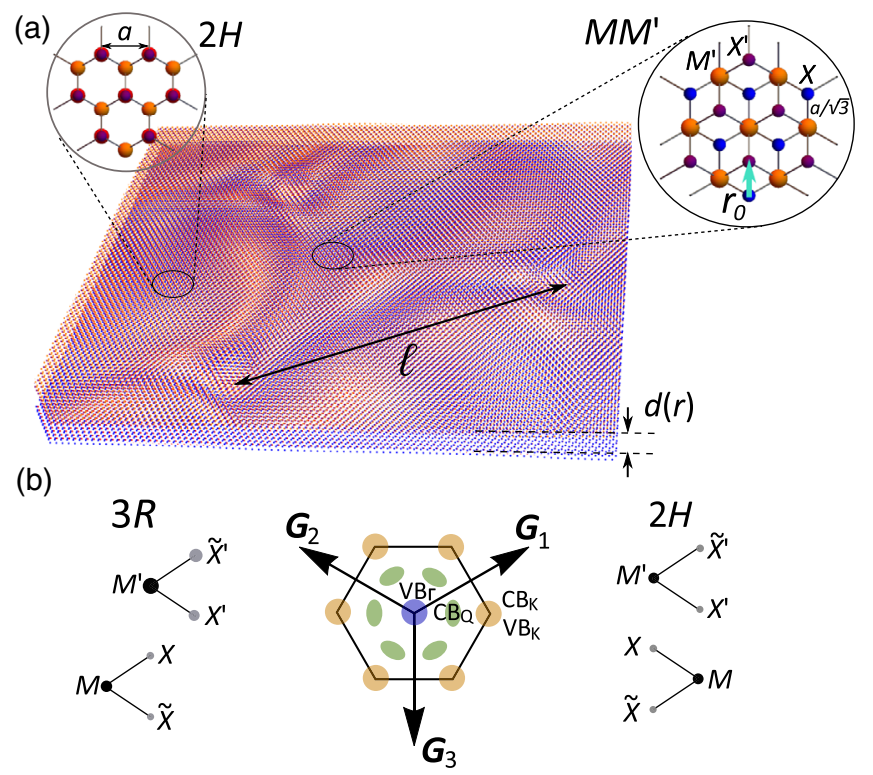

FIG. 1. (a) Sketch of lattice relaxation across moiré supercell of marginally twisted AP-bilayers $\left(\theta<\theta_{\mathrm{AP}}\right)$. Insets show local stacking configurations in $2 H$ domain and $M M^{\prime}$ seed. $X\left(X^{\prime}\right)$ and $M\left(M^{\prime}\right)$ label chalcogen and metal atoms, respectively, in the bottom (top) layer; (b) the left (right) panel reveals side view of $3 R-(2 H-)$ stacked TMD bilayers with the size of dots reflecting the layer asymmetry of the electronic states at the conduction band edge. Central panel: the first reciprocal lattice star and the first Brillouin zone of the TMD bilayers with marked conduction $\left(\mathrm{CB}_{Q}, \mathrm{CB}_{K}\right)$ and valence band $\left(\mathrm{VB}_{\Gamma}\right.$, and $\left.\mathrm{VB}_{K}\right)$ extrema.

overcome the system-size limitations of molecular dynamics simulations [26,28].

For adhesion energy, we use the form, $W_{\mathrm{P} / \mathrm{AP}}\left(\boldsymbol{r}_{0}, d\right)=$ $\sum_{n} f_{n}^{(\mathrm{P} / \mathrm{AP})}(d) e^{i \boldsymbol{G}_{n} \boldsymbol{r}_{0}}$, where $\boldsymbol{G}_{n}$ are the reciprocal lattice vectors of TMD. We truncate this sum at the first star of reciprocal lattice vectors, $\pm \boldsymbol{G}_{1,2,3},\left(\left|\boldsymbol{G}_{1,2,3}\right|=G\right.$, Fig. 1) and set $\boldsymbol{r}_{0}=0$ at the $X X^{\prime}$ stacking for both $\mathrm{P}$ and $\mathrm{AP}$ bilayers. This choice-together with the $D_{3 h}$ lattice symmetry of TMD monolayers-suggests [29] that $W_{\mathrm{P}}=f(d)+f_{1}(d) \sum_{n=1}^{3} \cos \left(\boldsymbol{G}_{n} \boldsymbol{r}_{0}\right)$ and $W_{\mathrm{AP}}=\tilde{f}(d)+$ $\sum_{n=1}^{3}\left[\tilde{f}_{1}(d) \cos \left(\boldsymbol{G}_{n} \boldsymbol{r}_{0}\right)+\tilde{g}_{1}(d) \sin \left(\boldsymbol{G}_{n} \boldsymbol{r}_{0}\right)\right]$. Then, we inspect the adhesion energies for various bilayers, computed using vdW DFT with the OPTB88 functional [30] implemented in QUANTUM ESPRESSO [43] for stacking configurations shown in Fig. 2. For P bilayers, the most energetically favorable are configurations $M X^{\prime}\left[\boldsymbol{r}_{0}=\right.$ $(0,-a / \sqrt{3})]$ and $X M^{\prime}\left[\boldsymbol{r}_{0}=(0, a / \sqrt{3})\right]$, which have equal energies [44] and correspond to twins of a $3 R$ bulk phase of a TMD. For AP bilayers, $2 H$ stacking $\left[\boldsymbol{r}_{0}=(0,-a / \sqrt{3})\right]$ has the lowest energy (in agreement with the $2 H$ bulk phase of these TMDs), rather than $M M^{\prime}$ stacking [configuration 5, $\boldsymbol{r}_{0}=(0, a / \sqrt{3})$ ] suggested in Ref. [27]. Configurations 6, $\boldsymbol{r}_{0}=-a / 3(1, \sqrt{3})$ and $\boldsymbol{r}_{0}=-a / 3(1,0)$, are such that $W_{\mathrm{P}}=f$ and $W_{\mathrm{AP}}=\tilde{f}$. The remaining two (2 and 4) have

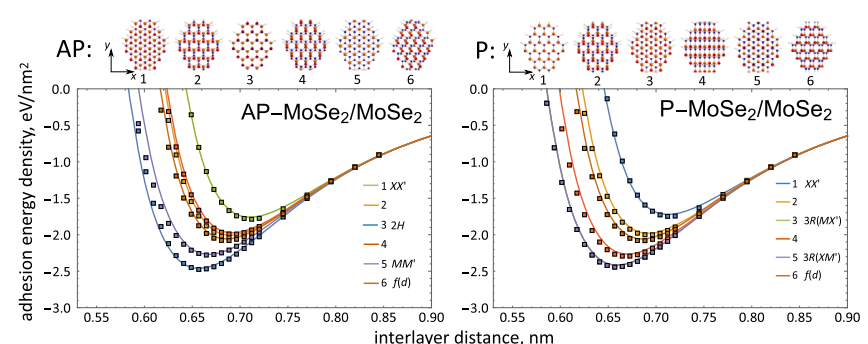

FIG. 2. VdW-DFT data for $\mathrm{MoSe}_{2} / \mathrm{MoSe}_{2}$ bilayers and their fits using $W_{\mathrm{P} / \mathrm{AP}}$ in Eq. (1) (for $\mathrm{WSe}_{2} / \mathrm{WSe}_{2}, \mathrm{MoS}_{2} / \mathrm{WS}_{2}$, $\mathrm{MoS}_{2} / \mathrm{MoS}_{2}, \mathrm{WS}_{2} / \mathrm{WS}_{2}$, and $\mathrm{MoS}_{2} / \mathrm{WS}_{2}$ bilayers see the Supplemental Material [30]). Fitting parameters are listed in Table S1 in the Supplemental Material [30].

offsets $\boldsymbol{r}_{0}=(0,-a / 2 \sqrt{3}) \quad\left[\boldsymbol{r}_{0}=(0, a / 2 \sqrt{3})\right]$ and $\boldsymbol{r}_{0}=$ $(0, a / 2 \sqrt{3})\left[\boldsymbol{r}_{0}=(0, a \sqrt{3} / 2)\right]$ for $\mathrm{AP}(\mathrm{P})$ bilayers.

Using the DFT data for bilayers shown in Fig. 2 and $\mathrm{S} 1$ in the Supplemental Material [30], we find the $d$ dependence of the factors $f, \tilde{f}, f_{1}, \tilde{f}_{1}$, and $\tilde{g}_{1}$, and plot them in Fig. S2. By inspection, we find that $f \approx \tilde{f}$ and $f_{1} \approx \tilde{f}_{1}+\tilde{g}_{1}$, over the broad interval of interlayer distances that covers the minima of $W_{\mathrm{P} / \mathrm{AP}}\left(\boldsymbol{r}_{0}, d\right)$, and, then, consider the following factors to make an informed choice of functions, $\tilde{f}, \tilde{f}_{1}$, and $\tilde{g}_{1}$. (i) Coulomb potential of a lattice of $\pm q$ ions, whose potential decays exponentially, $\sum_{n} \alpha_{n} e^{-G_{n} d} e^{i \boldsymbol{G}_{n} \boldsymbol{r}_{0}}$, with the distance from their plane $\left(\alpha_{0}=0\right.$ is due to the electroneutrality of each layer). This suggests a choice of $\tilde{g}_{1}=A_{2} e^{-G d}$ for the first star of reciprocal lattice vectors $\pm \boldsymbol{G}_{1,2,3}$. (ii) The overlap of atomic orbitals, strongest for chalcogen atoms in the outer (top and bottom) sublattices in each layer is determined by the exponential decay of atomic wave functions away from the plane, $|\psi(z)|^{2} \propto e^{-|z| / \rho}$. Also, tunneling between the layers is suppressed for electrons with a larger in-plane wave number [45], $\sum_{n} \beta_{n} e^{-d \sqrt{G_{n}^{2}+\rho^{-2}}} \cos \left(\boldsymbol{G}_{n} \boldsymbol{r}_{0}\right)$, so that we choose $\tilde{f}_{1}=A_{1} e^{-d \sqrt{G^{2}+\rho^{-2}}}$. (iii) Finally, following the earlier studies [46] of vdW interaction of TMDs, which has a long-distance asymptotic, $\propto-C / d^{4}$, we combine the short range repulsion and long-range $\mathrm{vdW}$ attraction into $\tilde{f}=-\sum_{n=1}^{3} C_{n} / d^{4 n}$. Then, we use

$$
\begin{aligned}
W_{\mathrm{P} / \mathrm{AP}}\left(\boldsymbol{r}_{0}, d\right)= & \sum_{n=1}^{3}\left[-\frac{C_{n}}{d^{4 n}}+A_{1} e^{-\sqrt{G^{2}+\rho^{-2}} d} \cos \left(\boldsymbol{G}_{n} \boldsymbol{r}_{0}\right)\right. \\
& \left.+A_{2} e^{-G d} \sin \left(\boldsymbol{G}_{n} \boldsymbol{r}_{0}+\varphi_{\mathrm{P} / \mathrm{AP}}\right)\right]
\end{aligned}
$$

with $\varphi_{\mathrm{P}}=\pi / 2, \varphi_{\mathrm{AP}}=0$, and fit the values of parameters $C_{1,2,3}, A_{1,2}, \rho$ to the DFT data listed in Table S1 in the Supplemental Material [30].

Lattice reconstruction in bilayers happens when energy gain from the formation of favorable stacking overcomes 
elastic energy cost of strain produced by the local mutual adjustment of the two lattices, $U=\sum_{l=t, b}\left[\left(\lambda_{l} / 2\right)\left(u_{i i}^{(l)}\right)^{2}+\right.$ $\left.\mu_{l}\left(u_{i j}^{(l)}\right)^{2}\right]$. Here, $\lambda_{t / b}, \quad \mu_{t / b}$, and $u_{i j}^{(t / b)}=\frac{1}{2}\left(\partial_{j} u_{i}^{(t / b)}+\right.$ $\left.\partial_{i} u_{j}^{(t / b)}\right)$, are the first Lamé coefficient, shear modulus, and strain tensors related to the local in-plane displacements in top and bottom layers. Values of Young's moduli and Poisson ratios determining $\lambda$ and $\mu$ coefficients for TMD crystals under consideration are listed in Table S3 in the Supplemental Material [30]. We neglect the energy cost of flexural deformations [47], see Sec. S3 in the Supplemental Material [30] allowing for the out-of-plane bending of the layers towards the optimal interlayer distance, $d_{\mathrm{P} / \mathrm{AP}}\left(\boldsymbol{r}_{0}\right)$, for each offset $\boldsymbol{r}_{0}$. We describe the latter by expanding $W_{\mathrm{P} / \mathrm{AP}}$ into Taylor series about the minimum point, $d_{0}$, of the zeroth Fourier harmonic term, $f(d) \approx f\left(d_{0}\right)+\varepsilon\left(d-d_{0}\right)^{2}$ (Table S1 in the Supplemental Material [30]), and, then, find $\boldsymbol{u}^{(t / b)}(\boldsymbol{r})$ that minimize energy,

$$
\begin{aligned}
\mathcal{E}= & \int d^{2} \boldsymbol{r}\left\{U-\varepsilon Z_{\mathrm{P} / \mathrm{AP}}^{2}\right. \\
& +\sum_{n=1}^{3}\left[A_{1} e^{-\sqrt{G^{2}+\rho^{-2}} d_{0}} \cos \left(\boldsymbol{g}_{n} \boldsymbol{r}+\boldsymbol{G}_{n}\left[\boldsymbol{u}^{(t)}-\boldsymbol{u}^{(b)}\right]\right)\right. \\
& \left.+A_{2} e^{-G d_{0}} \sin \left(\boldsymbol{g}_{n} \boldsymbol{r}+\boldsymbol{G}_{n}\left[\boldsymbol{u}^{(t)}-\boldsymbol{u}^{(b)}\right]+\varphi_{\mathrm{P} / \mathrm{AP})}\right]\right\} ; \\
Z_{\mathrm{P} / \mathrm{AP}}= & \left.\frac{1}{2 \varepsilon} \frac{\partial}{\partial d}\left[f(d)-W_{\mathrm{P} / \mathrm{AP}}(\boldsymbol{r}, d)\right]\right|_{d=d_{0}} .
\end{aligned}
$$

Here, $\boldsymbol{g}_{n}=\delta \boldsymbol{G}_{n}-\theta \hat{z} \times \boldsymbol{G}_{n}$ are reciprocal lattice vectors of moiré superlattice, which we also use to expand $\boldsymbol{u}^{(t / b)}(\boldsymbol{r})$ in Fourier harmonics up to the 80th reciprocal space star. Then, we minimize $\mathcal{E}$ with respect to those Fourier amplitudes numerically and obtain the displacements in each layer of the reconstructed bilayer. Using this method, we study moiré structure with $\theta^{\circ} \geq 0.2^{\circ}$.

Representative examples of lattice reconstructions in $\mathrm{P}$ and AP bilayers are shown in Fig. 3. For a larger angle, $\theta^{\circ}=4^{\circ}$ [Figs. 3(a) and 3(b)] adhesion-induced displacements are small and the two layers behave as rigid lattices. For $\theta^{\circ}<1^{\circ}$, twisted bilayers reconstruct into domain structures. For P bilayers [Fig. 3(d)], each reconstructed moiré supercell comprises two equal area triangular domains of $3 R\left(X M^{\prime} / M X^{\prime}\right)$ stacking, separated by partial dislocations, with $X X^{\prime}$ regions squeezed to the nodes on that partial dislocations network [Fig. 3(g)]. For AP bilayers [Fig. 3(c)], the reconstructed lattice features a honeycomb array of $2 \mathrm{H}$ domains separated by a dislocation network, where one half of the nodes are the seeds of the quasiequilibrium $M M^{\prime}$ phase (stacking configuration AP-5). As a quantitative measure for domain formation, we use a lateral distance, $\boldsymbol{\aleph}$, between the closest metal and sulfur atoms in top and bottom layers (for ideal $2 H$ and $3 R$

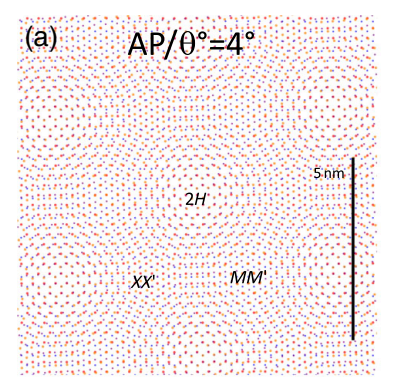

(b) $\mathrm{P} / 0^{\circ}=4^{\circ}$
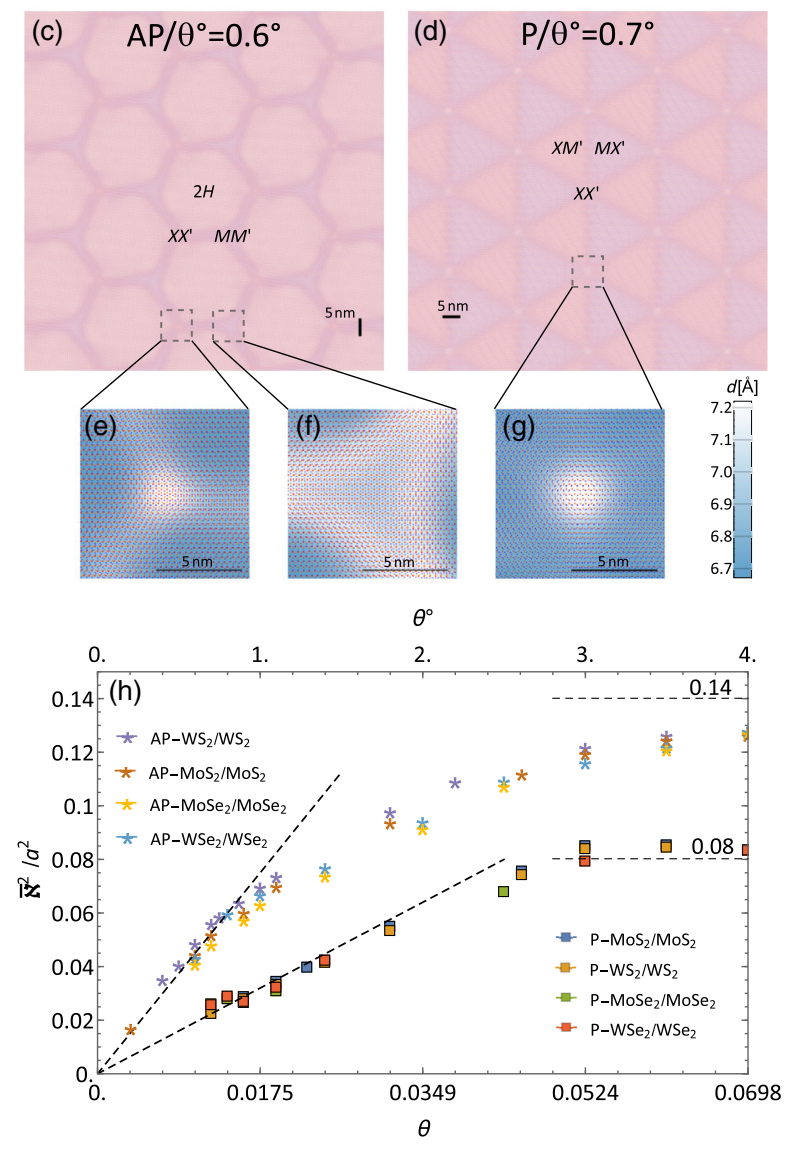

FIG. 3. (a)-(d) Reconstructed lattices of $\mathrm{WSe}_{2} / \mathrm{WSe}_{2} \mathrm{AP}(\mathrm{P})$ bilayer with $\theta^{\circ}=4^{\circ}>\theta_{\mathrm{AP} / \mathrm{P}}$ and $0.6^{\circ}\left(0.7^{\circ}\right)<\theta_{\mathrm{AP} / \mathrm{P}}$ twist (for $\theta^{\circ}=0.2^{\circ}$ see the Supplemental Material [30]). For $\theta>\theta_{\mathrm{AP} / \mathrm{P}}$ the two layers behave as rigid, for marginally twisted bilayers $(\theta \rightarrow 0) 2 H$ for $\mathrm{AP}$ and $3 R$ for $\mathrm{P}$ stacking domains emerge, separated by dislocations. (e)-(g) Intersections of dislocations, drawn over on the color maps of the varied interlayer distance. (h) Crossover from rigid twist to the fully developed domain structure in $\mathrm{P}$ and AP bilayers, quantified using average value of parameter $\overline{\aleph^{2}}$ described in the text.

domains, $\boldsymbol{\aleph}=0$ ) and compute its mean square, $\overline{\boldsymbol{\aleph}^{2}} / a^{2}$, over the supercell normalized by the TMD lattice constant, $a$. For rigidly rotated monolayers, $\overline{\aleph_{\mathrm{AP}}^{2}} \approx 0.14 a^{2}$ and $\overline{\aleph_{\mathrm{P}}^{2}} \approx$ $0.08 a^{2}$. Upon the formation of $2 H$ and $3 R$ domains, main contribution to $\overline{\aleph^{2}}$ comes from domain boundaries so that the asymptotic behavior, $\overline{\boldsymbol{N}}^{2} \propto 1 / \ell \propto \theta$, in Fig. 3(h) signals the formation of a domain structure at $\theta<\theta_{\mathrm{P}} \approx 0.044\left(2.5^{\circ}\right)$ 

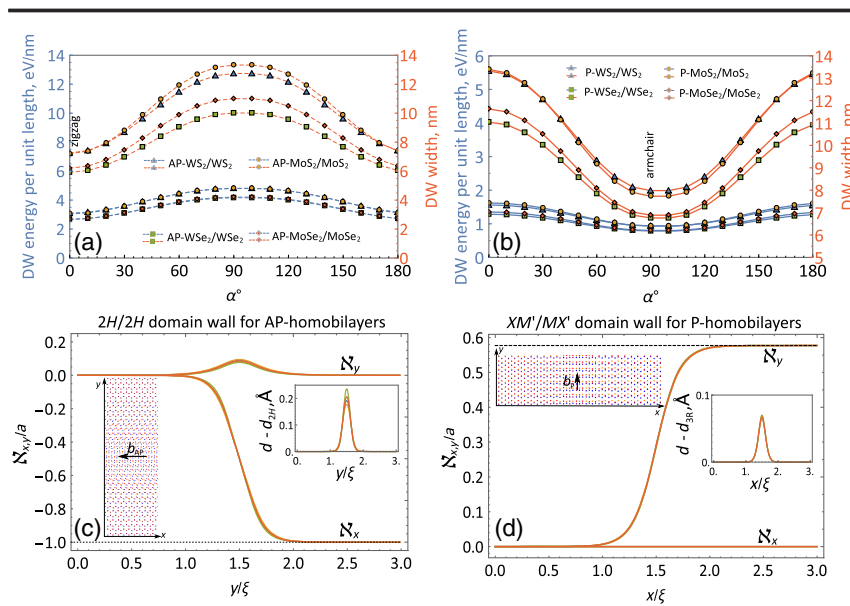

FIG. 4. (a),(b) Dependence of domain wall (DW) energy per unit length (blue) and width (red) on the angle between DW axis and zigzag direction ( $x$ axis) in TMD layer, $\alpha$, for AP and $\mathrm{P}$ bilayers, respectively. (c) and (d) describe DW (dislocation) profile for AP and $\mathrm{P}$ bilayers, respectively. The calculated profiles for various TMDs (shown by different colors) almost coincide. Here, $\xi=a \sqrt{\mu / 2 A_{1}} \exp \left[d_{0} \sqrt{G^{2}+\rho^{-2}} / 2\right]$.

and $\theta<\theta_{\mathrm{AP}} \approx 0.017\left(1^{\circ}\right)$. These quantitative estimates also explain why the molecular dynamics simulations performed in Refs. [26,28] for $\theta^{\circ}>3^{\circ}$ failed to establish the full picture of the lattice reconstruction in heterostructures of $2 \mathrm{D}$ crystals, having interpreted $M M^{\prime}$ areas in almost rigid AP bilayers [Fig. 3(a)], as fully developed domains. Note that superlattice patterns-perfect domains and the dislocation network - also appear in TMD heterobilayers with the same chalcogens $\left(\mathrm{WS}_{2} / \mathrm{MoS}_{2}\right.$ or $\mathrm{WSe}_{2} / \mathrm{MoSe}_{2}$ ), which have lattice mismatch $\delta \lesssim 0.3 \%$, whereas bilayers with $\theta>\theta_{\mathrm{P} / \mathrm{AP}}$, or $M \mathrm{~S}_{2} / M^{\prime} \mathrm{Se}_{2}$ heterostructures with $\delta \approx 3.8 \%$ behave as almost rigid crystals.

The formation of domain structures takes place when the energy gain from the expanded $2 H$ (for $\mathrm{AP}$ ) or $3 R$ (for $\mathrm{P}$ ) areas overcomes the energy cost of domain walls. The latter are nothing but dislocations: screw dislocations in $2 \mathrm{H}$ and partial screw dislocations in $3 R$ bilayers. The properties of such linear defects, analyzed using energy functional (2), are shown in Fig. 4. Here, we set $\theta=0$ in Eq. (2) and replace $\boldsymbol{u}^{t}-\boldsymbol{u}^{b}$ by $\boldsymbol{\aleph}$, such that $\boldsymbol{\aleph}(-\infty)=0$ and $\boldsymbol{\aleph}(+\infty)=$ $\boldsymbol{b}_{\mathrm{AP} / \mathrm{P}}$, where $\boldsymbol{b}_{\mathrm{AP}}=a(-1,0)$ is a Burgers vector of a dislocation in $2 H$ TMD $\left(b_{\mathrm{AP}}=a\right)$, and $\boldsymbol{b}_{\mathrm{P}}=a(0,1 / \sqrt{3})$ is a partial dislocation Burgers vector in $3 R$ bilayer $\left(b_{P}=a / \sqrt{3}\right)$. A vector $\boldsymbol{n}=(\cos \alpha, \sin \alpha)$ determines the orientation of the dislocation line with respect to zigzag axis in the crystal. We find (see Fig. 4) that the energy of a dislocation in $2 \mathrm{H}$ homobilayers is the lowest and the cross sectional width narrowest for a zigzag orientation of the defect line (screw dislocation); for $3 R$ homobilayers, the most favorable orientation of the partial dislocation axis is along the armchair direction (partial screw dislocation). These choices coincide with the orientations of domain boundaries shown in Fig. 3. In addition, in Fig. S8, we show the profile of edge dislocations that would form in perfectly aligned $(\theta<\delta)$ heterobilayers $M \mathrm{~S}_{2} / M^{\prime} \mathrm{S}_{2}$ and $M \mathrm{Se}_{2} / M^{\prime} \mathrm{Se}_{2}$ [48].

As a consequence of the domain formation, optoelectronic properties of marginally twisted bilayers would be dominated by the areas of $2 H$ and $3 R$ stacking. While $2 H$ bilayers have been deeply explored both theoretically [49-55] and experimentally [56-67], $3 R$ bilayers were studied less [57,64,68-76], despite that the latter have interesting features resulting from their lack of inversion symmetry. Table I gives the atomic decompositions of the states at the edges of conduction and valence bands, computed using DFT for $3 R$ bilayers (see the Supplemental Material for details [30]), highlighting the interlayer asymmetry of the states near band edges marked in Fig. 1(b). Such an asymmetry would make tunnelling characteristic of $M X^{\prime}$ and $X M^{\prime}$ domains different, leading to a manifestation of domain formation in conductive atomic force microscopy [77] as well as to a linear Stark shift, $\left[d_{z}\left(\mathrm{CB}_{Q}\right)-d_{z}\left(\mathrm{VB}_{\Gamma}\right)\right] E$, for the lowest-energy exciton transition in the bilayer, with the opposite sign of the shift in $M X^{\prime}$ and $M^{\prime} X$ stacking domains [78].

TABLE I. Orbital decomposition of DFT states at conduction $\left(\mathrm{CB}_{Q}\right)$ and valence $\left(\mathrm{VB}_{\Gamma}\right)$ band edges of $3 R$ homobilayers of $\mathrm{MX} 2$ [as labeled in Fig. 1(b)], the corresponding out-of-plane electric dipole moment, $d_{z}$, and ratio between tunneling currents into $M X^{\prime}$ and $X M^{\prime}$ stacking domains.

\begin{tabular}{lcrccccccc}
\hline \hline & $|\psi|^{2}[\%]$ & \multicolumn{1}{c}{$\tilde{X}$} & $M$ & $X$ & $X^{\prime}$ & $M^{\prime}$ & $\tilde{X}^{\prime}$ & $d_{z}(e \AA)$ & $I_{M X^{\prime}}: I_{X M^{\prime}}$ \\
\hline $\mathrm{MoS}_{2}$ & $\mathrm{CB}_{Q}$ & 4.2 & 30.6 & 3.3 & 5.5 & 49.6 & 6.8 & 0.73 & $1: 1.6$ \\
& $\mathrm{VB}_{\Gamma}$ & 10.6 & 41.8 & 0.9 & 0.7 & 36.0 & 10.0 & -0.20 & $1: 1$ \\
$\mathrm{MoSe}_{2}$ & $\mathrm{CB}_{Q}$ & 6.8 & 29.7 & 4.3 & 6.5 & 42.6 & 10.0 & 0.58 & $1: 1.6$ \\
& $\mathrm{VB}_{\Gamma}$ & 14.9 & 36.6 & 1.2 & 1.0 & 31.9 & 14.3 & -0.17 & $1: 1$ \\
$\mathrm{WS}_{2}$ & $\mathrm{CB}_{Q}$ & 4.3 & 28.6 & 4.0 & 6.8 & 48.9 & 7.4 & 0.81 & $1: 1.7$ \\
& $\mathrm{VB}_{\Gamma}$ & 10.3 & 41.5 & 1.6 & 1.3 & 35.5 & 9.9 & -0.21 & $1: 1$ \\
\multirow{2}{*}{$\mathrm{WSe}_{2}$} & $\mathrm{CB}_{Q}$ & 7.0 & 24.4 & 6.2 & 10.3 & 40.6 & 11.5 & 0.80 & $1: 1.6$ \\
& $\mathrm{VB}_{\Gamma}$ & 14.5 & 36.3 & 2.2 & 1.8 & 31.4 & 13.7 & -0.19 & $1: 1$ \\
\hline \hline
\end{tabular}




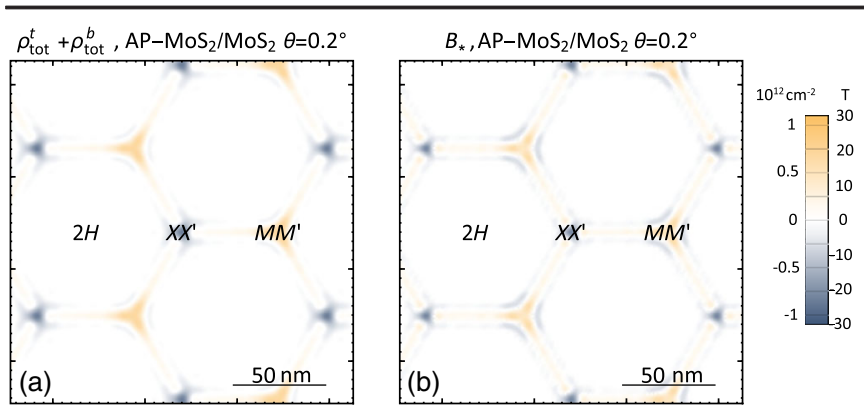

FIG. 5. (a),(b) Distribution of piezoelectric charge density and pseudomagnetic field induced in AP bilayers $\mathrm{MoS}_{2} / \mathrm{MoS}_{2}$ $\left(\theta=0.2^{\circ}\right)$.

Lacking inversion symmetry, TMD monolayers are piezoelectric crystals. The inhomogeneous strain concentrated in each layer at domain walls results in piezocharges with density, $\rho_{\text {piezo }}^{(t / b)}=e_{11}\left[2 \partial_{x} u_{x y}^{(t / b)}+\partial_{y}\left(u_{x x}^{(t / b)}-u_{y y}^{(t / b)}\right)\right]$. In $\mathrm{P}$ bilayers, the opposite signs of strain fields in the top and bottom layers leads to the mutual compensation of their piezoelectric charges. In AP bilayers the inversion of the sign $e_{11}^{t}=-e_{11}^{b}$ of piezoparameter in the two layers adds up charges, to the values shown in Fig. 5(a), computed after taking into account partial screening of piezoelectric field by dielectric polarizability of TMD layers (see in the Supplemental Material [30] for details), with opposite signs in $M M^{\prime}$ to $X X^{\prime}$ corners of the domain wall network. Then, the $M M^{\prime}$ regions and $2 H / 2 H$ DWs will attract electrons in $n$-doped AP homobilayers, giving rise to a larger tunnel current response than inside the $2 H$ domains [77]. These spots of piezocharges, with the values up to $\sim 10^{12} \mathrm{~cm}^{-2}$, can be viewed as "quantum dots" for electrons and holes in the vicinity of the band edges of the bilayers. The same areas are also the hot spots of pseudomagnetic fields $\left[B_{*} \propto \rho_{\text {piezo }}^{(t / b)}\right.$, Fig. 5(b)], generated by inhomogeneous strain for electrons in multivalley semiconductors [79], which makes the dislocation network sites $\left(M M^{\prime}\right.$ and $X X^{\prime}$ regions) interesting objects for optoelectronic studies.

We thank R. Gorbachev, S. Haigh, and H. Park for fruitful discussions. This work has been supported by EPSRC Grants No. EP/S019367/1, No. EP/S030719/1, and No. EP/N010345/1, ERC Synergy Grant Hetero2D, Lloyds Register Foundation Nanotechnology grant, European Graphene Flagship Project, and EU Quantum Technology Flagship Project No. 2D-SIPC.

[1] K. Novoselov, A. Mishchenko, A. Carvalho, and A. C. Neto, Science 353, aac9439 (2016).

[2] L. A. Ponomarenko, R. V. Gorbachev, G. L. Yu, D. C. Elias, R. Jalil, A. A. Patel, A. Mishchenko, A. S. Mayorov, C. R. Woods, J. R. Wallbank et al., Nature (London) 497, 594 (2013).
[3] C. R. Dean, L. Wang, P. Maher, C. Forsythe, F. Ghahari, Y. Gao, J. Katoch, M. Ishigami, P. Moon, M. Koshino et al., Nature (London) 497, 598 (2013).

[4] N. Y. Kim, H. Y. Jeong, J. H. Kim, G. Kim, H. S. Shin, and Z. Lee, ACS Nano 11, 7084 (2017).

[5] M. Lee, J. R. Wallbank, P. Gallagher, K. Watanabe, T. Taniguchi, V. I. Fal'ko, and D. Goldhaber-Gordon, Science 353, 1526 (2016).

[6] M. Yankowitz, J. Xue, D. Cormode, J. D. SanchezYamagishi, K. Watanabe, T. Taniguchi, P. Jarillo-Herrero, P. Jacquod, and B. J. LeRoy, Nat. Phys. 8, 382 (2012).

[7] Y. Cao, V. Fatemi, S. Fang, K. Watanabe, T. Taniguchi, E. Kaxiras, and P. Jarillo-Herrero, Nature (London) 556, 43 (2018).

[8] Y. Cao, V. Fatemi, A. Demir, S. Fang, S. L. Tomarken, J. Y. Luo, J. D. Sanchez-Yamagishi, K. Watanabe, T. Taniguchi, E. Kaxiras et al., Nature (London) 556, 80 (2018).

[9] M. Yankowitz, S. Chen, H. Polshyn, Y. Zhang, K. Watanabe, T. Taniguchi, D. Graf, A. F. Young, and C. R. Dean, Science 363, 1059 (2019).

[10] S. G. Xu, A. I. Berdyugin, P. Kumaravadivel, F. Guinea, R. Krishna Kumar, D. A. Bandurin, S. V. Morozov, W. Kuang, B. Tsim, S. Liu et al., Nat. Commun. 10, 4008 (2019).

[11] J. S. Alden, A. W. Tsen, P. Y. Huang, R. Hovden, L. Brown, J. Park, D. A. Muller, and P. L. McEuen, Proc. Natl. Acad. Sci. U.S.A. 110, 11256 (2013).

[12] H. Yoo, R. Engelke, S. Carr, S. Fang, K. Zhang, P. Cazeaux, S. H. Sung, R. Hovden, A. W. Tsen, T. Taniguchi et al., Nat. Mater. 18, 448 (2019).

[13] S. Zhou, J. Han, S. Dai, J. Sun, and D. J. Srolovitz, Phys. Rev. B 92, 155438 (2015).

[14] F. Gargiulo and O. V. Yazyev, 2D Mater. 5, 015019 (2017).

[15] M. Van Wijk, A. Schuring, M. Katsnelson, and A. Fasolino, 2D Mater. 2, 034010 (2015).

[16] S. Dai, Y. Xiang, and D. J. Srolovitz, Nano Lett. 16, 5923 (2016).

[17] N. N. T. Nam and M. Koshino, Phys. Rev. B 96, 075311 (2017).

[18] A. Kerelsky, C. Rubio-Verd, L. Xian, D. M. Kennes, D. Halbertal, N. Finney, L. Song, S. Turkel, L. Wang, K. Watanabe et al., arXiv:1911.00007.

[19] J. Kunstmann, F. Mooshammer, P. Nagler, A. Chaves, F. Stein, N. Paradiso, G. Plechinger, C. Strunk, C. Schüller, G. Seifert et al., Nat. Phys. 14, 801 (2018).

[20] P. Rivera, H. Yu, K. L. Seyler, N. P. Wilson, W. Yao, and X. $\mathrm{Xu}$, Nat. Nanotechnol. 13, 1004 (2018).

[21] P. K. Nayak, Y. Horbatenko, S. Ahn, G. Kim, J.-U. Lee, K. Y. Ma, A.-R. Jang, H. Lim, D. Kim, S. Ryu et al., ACS Nano 11, 4041 (2017).

[22] K. L. Seyler, P. Rivera, H. Yu, N. P. Wilson, E. L. Ray, D. G. Mandrus, J. Yan, W. Yao, and X. Xu, Nature (London) 567, 66 (2019).

[23] K. Tran, G. Moody, F. Wu, X. Lu, J. Choi, K. Kim, A. Rai, D. A. Sanchez, J. Quan, A. Singh et al., Nature (London) 567, 71 (2019).

[24] C. Jin, E. C. Regan, A. Yan, M. I. B. Utama, D. Wang, S. Zhao, Y. Qin, S. Yang, Z. Zheng, S. Shi et al., Nature (London) 567, 76 (2019). 
[25] E. M. Alexeev, D. A. Ruiz-Tijerina, M. Danovich, M. J. Hamer, D. J. Terry, P. K. Nayak, S. Ahn, S. Pak, J. Lee, J. I. Sohn et al., Nature (London) 567, 81 (2019).

[26] M. H. Naik and M. Jain, Phys. Rev. Lett. 121, 266401 (2018).

[27] S. Carr, D. Massatt, S. B. Torrisi, P. Cazeaux, M. Luskin, and E. Kaxiras, Phys. Rev. B 98, 224102 (2018).

[28] M. H. Naik, I. Maity, P. K. Maiti, and M. Jain, J. Phys. Chem. C 123, 9770 (2019).

[29] This is because aligned P-oriented TMD bilayers with opposite in-plane offsets represent mirror copies of each other resulting in the same interaction value for $\pm \boldsymbol{r}_{0}$; whereas for AP orientation, the lack of inversion symmetry in each layer allows for both even and odd terms.

[30] See the Supplemental Material at http://link.aps.org/ supplemental/10.1103/PhysRevLett.124.206101 for details of DFT calculations, values of fitted parameters, and piezocharge density calculations, which includes Refs. [3142].

[31] S.-Y. Chen, C. Zheng, M. S. Fuhrer, and J. Yan, Nano Lett. 15, 2526 (2015).

[32] M.-L. Lin, Q.-H. Tan, J.-B. Wu, X.-S. Chen, J.-H. Wang, Y.-H. Pan, X. Zhang, X. Cong, J. Zhang, W. Ji et al., ACS Nano 12, 8770 (2018).

[33] X. Zhang, W. P. Han, J. Wu, S. Milana, Y. Lu, Q. Q. Li, A. C. Ferrari, and P. H. Tan, Phys. Rev. B 87, 115413 (2013).

[34] G. Kresse and J. Furthmüller, Phys. Rev. B 54, 11169 (1996).

[35] W. Schutte, J. De Boer, and F. Jellinek, J. Solid State Chem. 70, 207 (1987).

[36] K. Bronsema, J. De Boer, and F. Jellinek, Z. Anorg. Allg. Chem. 540, 15 (1986).

[37] L. D. Landau and E. M. Lifshitz, Theory of Elasticity (3rd ed), Course of Theoretical Physics (Elsevier, Oxford, 1986).

[38] N. Iguiñiz, R. Frisenda, R. Bratschitsch, and A. CastellanosGomez, Adv. Mater. 31, 1807150 (2019).

[39] C. Androulidakis, K. Zhang, M. Robertson, and S. Tawfick, 2D Mater. 5, 032005 (2018).

[40] K.-A. N. Duerloo, M. T. Ong, and E. J. Reed, J. Phys. Chem. Lett. 3, 2871 (2012).

[41] H. Zhu, Y. Wang, J. Xiao, M. Liu, S. Xiong, Z. J. Wong, Z. Ye, Y. Ye, X. Yin, and X. Zhang, Nat. Nanotechnol. 10, 151 (2015).

[42] A. Laturia, M. L. Van de Put, and W. G. Vandenberghe, npj 2D Mater. Appl. 2, 6 (2018).

[43] P. Giannozzi, S. Baroni, N. Bonini, M. Calandra, R. Car, C. Cavazzoni, D. Ceresoli, G. L. Chiarotti, M. Cococcioni, I. Dabo et al., J. Phys. Condens. Matter 21, 395502 (2009).

[44] For $\mathrm{MoSe}_{2} / \mathrm{WSe}_{2}$ (Fig. 2) and $\mathrm{MoS}_{2} / \mathrm{WS}_{2}$ [Figs. S1(e) and $\mathrm{S} 1(\mathrm{f})$ in the Supplemental Material [30]] heterobilayers energies of twins $\left(M X^{\prime}\right.$ and $\left.X M^{\prime}\right)$ of $3 R$-stacking configuration are almost the same.

[45] R. Bistritzer and A. H. MacDonald, Proc. Natl. Acad. Sci. U.S.A. 108, 12233 (2011).

[46] J. F. Dobson, A. White, and A. Rubio, Phys. Rev. Lett. 96, 073201 (2006).

[47] Up to room temperature the variation of interlayer distances, $\sqrt{\left\langle\delta d^{2}\right\rangle_{T}}$, due to thermal out-of-plane vibrations is by an order of magnitude less than the difference, $\left|d_{2 H}-d_{0}\right|$, between the interlayer distance for $2 \mathrm{H}$ stacking and its value averaged over stacking configurations present in a moire supercell (see the Supplemental Material [30], Section S4). In fact, we use the estimated value $\sqrt{\left\langle\delta d^{2}\right\rangle_{300 \mathrm{~K}}} \approx 4 \times$ $10^{-3} \mathrm{~nm}$ for the size of the symbols showing the DFTcomputed adhesion energies in Fig. 2 to point out that the following analysis is applicable to all $T \leq 300 \mathrm{~K}$.

[48] The described domain structures and dislocation networks result from the fabrication-induced twist in a bilayer. As such, they are long-living quasiequilibrium states, which may slowly relax upon annealing by dislocations escaping through the sample edges, resulting in larger-size domains of perfect $3 R$ (for $\mathrm{P}$ ) and $2 H$ (for $\mathrm{AP}$ ) stackings.

[49] T. Cheiwchanchamnangij and W. R. L. Lambrecht, Phys. Rev. B 85, 205302 (2012).

[50] L. Debbichi, O. Eriksson, and S. Lebègue, Phys. Rev. B 89, 205311 (2014).

[51] D. A. Ruiz-Tijerina, M. Danovich, C. Yelgel, V. Zólyomi, and V. I. Fal'ko, Phys. Rev. B 98, 035411 (2018).

[52] J. E. Padilha, H. Peelaers, A. Janotti, and C. G. Van de Walle, Phys. Rev. B 90, 205420 (2014).

[53] T.-R. Chang, H. Lin, H.-T. Jeng, and A. Bansil, Sci. Rep. 4, 6270 (2015).

[54] S. Fang, R. Kuate Defo, S. N. Shirodkar, S. Lieu, G. A. Tritsaris, and E. Kaxiras, Phys. Rev. B 92, 205108 (2015).

[55] Z. Gong, G.-B. Liu, H. Yu, D. Xiao, X. Cui, X. Xu, and W. Yao, Nat. Commun. 4, 2053 (2013).

[56] K. F. Mak, C. Lee, J. Hone, J. Shan, and T. F. Heinz, Phys. Rev. Lett. 105, 136805 (2010).

[57] R. Suzuki, M. Sakano, Y. Zhang, R. Akashi, D. Morikawa, A. Harasawa, K. Yaji, K. Kuroda, K. Miyamoto, T. Okuda et al., Nat. Nanotechnol. 9, 611 (2014).

[58] A. Chernikov, C. Ruppert, H. M. Hill, A. F. Rigosi, and T. F. Heinz, Nat. Photonics 9, 466 (2015).

[59] A. M. Jones, H. Yu, J. S. Ross, P. Klement, N. J. Ghimire, J. Yan, D. G. Mandrus, W. Yao, and X. Xu, Nat. Phys. 10, 130 (2014).

[60] S. Wu, J. S. Ross, G.-B. Liu, G. Aivazian, A. Jones, Z. Fei, W. Zhu, D. Xiao, W. Yao, D. Cobden et al., Nat. Phys. 9, 149 (2013).

[61] T. Jiang, H. Liu, D. Huang, S. Zhang, Y. Li, X. Gong, Y.-R. Shen, W.-T. Liu, and S. Wu, Nat. Nanotechnol. 9, 825 (2014).

[62] I. G. Lezama, A. Arora, A. Ubaldini, C. Barreteau, E. Giannini, M. Potemski, and A. F. Morpurgo, Nano Lett. 15, 2336 (2015).

[63] B. Fallahazad, H. C. P. Movva, K. Kim, S. Larentis, T. Taniguchi, K. Watanabe, S. K. Banerjee, and E. Tutuc, Phys. Rev. Lett. 116, 086601 (2016).

[64] R. Akashi, M. Ochi, S. Bordács, R. Suzuki, Y. Tokura, Y. Iwasa, and R. Arita, Phys. Rev. Applied 4, 014002 (2015).

[65] I. C. Gerber, E. Courtade, S. Shree, C. Robert, T. Taniguchi, K. Watanabe, A. Balocchi, P. Renucci, D. Lagarde, X. Marie et al., Phys. Rev. B 99, 035443 (2019).

[66] R. Pisoni, T. Davatz, K. Watanabe, T. Taniguchi, T. Ihn, and K. Ensslin, Phys. Rev. Lett. 123, 117702 (2019).

[67] A. Arora, M. Koperski, A. Slobodeniuk, K. Nogajewski, R. Schmidt, R. Schneider, M. R. Molas, S. M. de Vasconcellos, 
R. Bratschitsch, and M. Potemski, 2D Mater. 6, 015010 (2018).

[68] A. Kormányos, V. Zólyomi, V. I. Fal'ko, and G. Burkard, Phys. Rev. B 98, 035408 (2018).

[69] X. Zhang, W.-Y. Shan, and D. Xiao, Phys. Rev. Lett. 120, 077401 (2018).

[70] J. Yan, J. Xia, X. Wang, L. Liu, J.-L. Kuo, B. K. Tay, S. Chen, W. Zhou, Z. Liu, and Z. X. Shen, Nano Lett. 15, 8155 (2015).

[71] N. Ubrig, S. Jo, M. Philippi, D. Costanzo, H. Berger, A. B. Kuzmenko, and A.F. Morpurgo, Nano Lett. 17, 5719 (2017).

[72] Y. Wang, C. Cong, J. Shang, M. Eginligil, Y. Jin, G. Li, Y. Chen, N. Peimyoo, and T. Yu, Nanoscale Horizons 4, 396 (2019).

[73] J. Van Baren, G. Ye, J.-A. Yan, Z. Ye, P. Rezaie, P. Yu, Z. Liu, R. He, and C. H. Lui, 2D Mater. 6, 025022 (2019).
[74] J. Shi, P. Yu, F. Liu, P. He, R. Wang, L. Qin, J. Zhou, X. Li, J. Zhou, X. Sui et al., Adv. Mater. 29, 1701486 (2017).

[75] E. Mishina, N. Sherstyuk, S. Lavrov, A. Sigov, A. Mitioglu, S. Anghel, and L. Kulyuk, Appl. Phys. Lett. 106, 131901 (2015).

[76] M. Zhao, Z. Ye, R. Suzuki, Y. Ye, H. Zhu, J. Xiao, Y. Wang, Y. Iwasa, and X. Zhang, Light Sci. Appl. 5, e16131 (2016).

[77] A. Weston, Y. Zou, V. Enaldiev, A. Summerfield, N. Clark, V. Z'olyomi, A. Graham, C. Yelgel, S. Magorrian, M. Zhou et al., arXiv:1911.12664.

[78] J. Sung, Y. Zhou, G. Scuri, V. Zólyomi, T. I. Andersen, H. Yoo, D. S. Wild, A. Y. Joe, R. J. Gelly, H. Heo et al., arXiv:2001.01157.

[79] S. Iordanskii and A. Koshelev, Pis'ma Zh. Eksp. Teor. Fiz. 41, 471 (1985). 